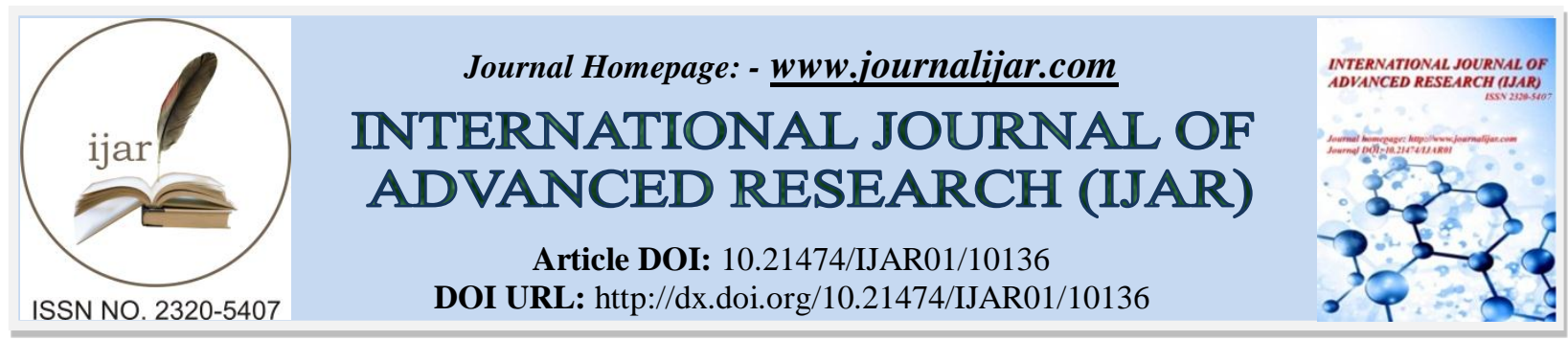

RESEARCH ARTICLE

\title{
EVALUATION OF IMMUNOMODULATORY EFFECT OF FILARIAL ANTIGENS ON THE DEVELOPMENT OF TYPE I DIABETES.
}

Manjusha Hivre and Sameer Khan.

1. Assistant Professor, Department of Biochemistry, MGM Medical College Aurangabad.

2. Deputy Medical Superitendent, WCL Pench Area, Chhindwara, Madhya Pradesh.

\section{Manuscript Info}

Manuscript History

Received: 01 October 2019

Final Accepted: 03 November 2019

Published: December 2019

Key words:-

Hygiene Hypothesis,

Immunomodulatory, Filarial Proteins,

Type 1 Diabetes, Autoimmune Disorder.

\begin{abstract}
The rapid worldwide increase of this disease has challenged the researchers for explaining the rising trend in type 1 diabetes. Evidences are available in support of an inverse relationship between worm infection and $\mathrm{T}$ helper type 1/17 (Th1/17) - based inflammatory disorder such as Type 1 diabetes suggesting the immunomodulatory effect of helminth molecules in this condition. The objective of the study was to evaluate the immunomodulatory effect of filarial antigens (Bm mf's/ Bmmf'ES'/ rBmCys) on the development of type 1 diabetes. The mice were either pre-treated with or without the said proteins using alum as adjuvant $(25 \mu \mathrm{g})$ followed by experimental induction of diabetes for 2 months. The blood glucose level and pancreatic histopathological changes were measured. There was significant reduction in the glucose level in diabetic group of mice pre-treated separately with filarial native proteins (Bm $\mathrm{mf}$ 's and Bmmf'ES) compared to diabetic mice pre-treated with only $\operatorname{Alum}(\mathrm{p}<0.002$ andp $<0.001$ ). Almost $70 \%$ of the mice pre-treated with Bmmf'ES showed recovery from diabetes at the end of the experiment period. Although all mice had evidence of ongoing pancreatic islet cell inflammation by histology, mice pre-treated with filarial proteins had greater numbers of total intact islets and non-infiltrated islets than untreated group of mice. These findings suggest that filarial derived proteins play pivotal role in the amelioration of disease condition in mice and act as novel immunomodulators in the treatment of type 1 diabetes.
\end{abstract}

Copy Right, IJAR, 2019,. All rights reserved.

\section{Introduction:-}

Epidemiological data from the World Health Organization (WHO) largely supports "hygiene hypothesis", indicating that westernized countries are facing alarming increase in childhood allergic conditions like rhinitis, atopic dermatitis and asthma (Patel et al., 2008), inflammatory bowel diseases (IBD) and autoimmune disorders like type 1 diabetes, multiple sclerosis and rheumatoid arthritis (Weinstock and Elliot, 2009). In contrast, several autoimmune disorders have reduced incidence and severity in geographical regions with high parasite load (Sewell et al., 2002). In tune with these observations, it has been suggested that bringing up children in extremely hygienic environments may have negative impact on their immune development making them susceptible to autoimmune and allergic disorders later in life (Maizels et al., 2009).

Corresponding Author:-Manjusha Hivre.

Address:- Assistant Professor, Department of Biochemistry, MGM Medical College Aurangabad. 
The parasitic infections might attenuate the host immune system to be more tolerant and avoid exacerbated inflammatory response. The helminth parasites thus could be a rich source of immunomodulators with potential therapeutic value for these diseases (Harnett et al., 2003). In a bidirectional relationship parasites develop strategies, including mechanisms to escape detection and active manipulation of hosts' immune cells, to circumvent or dampen the host response(s). This ability to interfere with the host's immune responses affords parasites the opportunity to establish, develop, reproduce and complete their life cycles.

Type 1Diabetes (T1D) is a chronic autoimmune disorder characterized by a progressive cellular infiltration of the pancreas resulting in the destruction of insulin-producing $\beta$-islets cells (Tisch and McDevitt, 1996). It manifests as a chronic inflammatory response involving islet infiltration (insulitis) by lymphocytes and monocytes resulting in destruction of majority of $\beta$ cells. Reportedly, 37 epidemiological studies conducted from 1960 to 1996 suggested that there has been an increase in the incidence of type 1 diabetes worldwide by $3 \%$ per year during this period (Onkamo et al., 1999). Environmental change responsible for the recent increase in autoimmune diseases like T1D is the loss of chronic parasitic infections in developed countries. Also persons infected with chronic parasitic worm infections are found to have lower rates of autoimmune diseases than others living in the same environment (Fleming and Cook, 2006).

Experimentally, a number of helminth parasites, including infection with a tissue-invasive filarial nematode, Litomosoides sigmodontis have prevented the onset of type 1 diabetes in NOD mice by altering the levels of IFN- $\gamma$ and IL-10 (Hubner et al., 2009). Also the nematodes H. polygyrus and T. spiralis are found to play vital role in the prevention of onset or suppression of severity of type 1 diabetes in non-obese diabetic (NOD) mice (Saunders et al.,2007).

Multiple studies conducted in India have found that individuals infected with chronic filarial parasitic worm infections have lower rates of type 1 diabetes than others living in the same environment (Aravindhan et al., 2010; Ramachandran et al.,1996). The infected host's immune response to these worms is characterized by reduced production of IFN- $\gamma$ / TNF- $\alpha$ and increased production of IL-4 / IL-10 together with elevated IgG4 antibody levels against parasite antigens suggesting a predominant Th2 / anti-inflammatory phenotype conducive to both parasite survival and host health (McInnes et al., 2003 ; Harnett et al., 2004). Filarial nematode Acanthocheilonema viteae, derived excretory secretory (ES) phosphorylcholine-containing glycoprotein, ES-62 of adult stage has been shown to inhibit the development of allergic responses and suppression of the LPS- induced rheumatoid arthritis (McInnes et al., 2003).

Cystatins are natural, tight-binding cysteine protease inhibitors that are involved in parasite-induced immunomodulation, as it induces cellular hypo-reactivity of T cells, modulates the production of anti-inflammatory cytokines, and down regulate essential co stimulatory molecules on macrophages (Hartmann et al., 1997; Schierack et al., 2003). They have also shown to account for a major proportion of the immunosuppressive activity of secreted filarial proteins and therefore is suggested to be considered as a major pathogenicity factor of filariae (Hartmann and Lucius, 2003). Hence this mechanism of immunomodulation by filarial cystatin may be exploited to create new antiinflammatory therapy against type 1 diabetes.

In view of the above considerations the present study was planned to assess the immunomodulatory effect of filarial proteins viz. Brugia malayi Cystatin (rBmCys), microfilarial excretory-secretory (Bm mf ES) and microfilarial soluble (Bm $\mathrm{mf} \mathrm{S}$ ) antigens in type 1 diabetes using mouse model.

\section{Material and Methods:-}

\section{Experimental Animals and B. malayi Parasites:}

After getting clearance from the Institutional Animal Ethics Committee, BALB/c mice (of 8-10 weeks of age and weighing $2530 \mathrm{~g}$ ) bred and maintained in the animal house of the institute were used in this study as per the guidelines of Committee for the Purpose of Control and Supervision of Experiments on Animals, Government of India (CPCSEA). The animals were maintained under standard laboratory conditions with free access to animal chow and drinking water ad libitum and all the surgical procedures were performed under the strict aseptic conditions. 
Micro-filariea were obtained by lavage of the peritoneal cavities of jirds with intraperitoneal filarial infection of 3 months or more duration. Brugia malayi infective stage (L3) larvae used in this study were obtained using Baermann's technique.

Filarial native antigens (Bm mf ES \& Bm mf S):

Bm mf ES antigen was prepared as described by (Chenthamarakshan e t al.,1996). Bm mf S antigen was prepared as described by (Kaliraj et al. 1982) with few modifications.

Recombinant Cystatin antigen (rBmCys):

rBmCys antigen was expressed and purified. The recombinant gene constructs pRSETA-BmCys was maintained in Top 10F' E.coli host. E.coli BL21(DE3) pLysS was transformed with the desired gene construct in pRSET-A. A single colony of fresh transformant was inoculated into $1.5 \mathrm{ml} \mathrm{LB}$ and grown overnight. 1M IPTG was added for the expression of recombinant protein. Collected supernatant was passed through immobilized cobalt metal affinity column chromatography (Clontech, Mountain View, CA) to purify the His tagged recombinant proteins. The expression pattern and purity were analysed by 15\% SDS-PAGE. Presence of Histidine-tag in the purified protein was detected using penta His-HRP monoclonal antibodies (Qiagen Valencia, CA). Concentration of protein was estimated by micro BCA method (Thermo Fisher).

Induction and assessment of Multiple Low-Dose Streptozotocin-induced Diabetes (MLDS): Type I diabetes was induced in BALB/c mice using low dose Streptozotocin (STZ) protocol (40 mg STZ / Kg/ day, injected intraperitoneally for five consecutive days) as described by Santos20Junior et al .

\section{Prophylactic treatment of mice with filarial proteins followed by STZ induction of diabetes:}

To assess the effect of prophylactic treatment with filarial proteins on development of T1D, mice in 5 groups were pre-treated with $\mathrm{rBmCys} / \mathrm{Bm} \mathrm{mf} \mathrm{ES} / \mathrm{Bm} \mathrm{mf} \mathrm{S}$ antigens followed by induction of T1D using STZ. Appropriate control groups of mice with equal number of animals were also maintained and followed up for 7 weeks.

STZ-rBmCys:

Injected with rBmCys at the intervals of 15 days for 2 months followed by STZ induction of diabetes.

STZ-Bm mf ES:

Injected with Bm mf ES antigen at the intervals of 15 days for 2 months followed by STZ induction of diabetes

STZ-Bm mf S:

Injected with Bm mf S antigen at the intervals of 15 days for 2 months followed by STZ induction of diabetes

\section{STZ-Alum:}

Injected with only alum adjuvant followed by STZ induction of diabetes.

STZ:

STZ Induction of diabetes (control group).

Analysis of immunomodulatory effect of filarial antigens on mice with type I diabetes:

1. In vitro proliferation assay of spleen cells by modified MTT cell viability method: After $72 \mathrm{hr}$ incubation, the extent of cell proliferation was measured by modified MTT cell viability assay i.e. MTS method using Cell Titer $96 \mathrm{R}$ aqueous nonradioactive cell proliferation assay (Promega, USA).

2. Analysis of cytokines by ELISA: Supernatants collected from the spleen cell cultures were used for measuring IL-10, IL-4, IL5, IFN- $\gamma$ and TNF- $\alpha$ by two site sandwich Enzyme Immuno assay (Invitrogen, USA) as per the protocol supplied by the manufacturer.

3. Monitering of diabetes status in mice: The mice were monitored for fasting glucose levels in blood using standard glucometer (Gluco Chek, Major Biochemical Corporation, Taiwan). Mice with blood glucose level of $\geq 200 \mathrm{mg} / \mathrm{dl}$ in samples for two consecutive weeks were considered as diabetic.

4. Anti-insulin antibodies in mice sera: Serum from all the above experimental and control groups of mice were collected to evaluate the anti-insulin antibodies (IgG, IgG1, IgG2a and IgM Abs) using standard enzyme-linked immunosorbent assays (ELISA). 
5. Histological assessment of pancreas inflammation: Total islets were counted and the changes looked for in each group were islets morphology number of islets, inflammation, lymphocyte infiltration. Numbers of islets of four longitudinal sections of each pancreas were assessed. The severity of insulitis was scored as non-infiltrated , peri-insulitis or intra-insulitis.

\section{Statistical analysis:-}

Results for various parameters are presented as mean \pm standard error of the means (SEM). The tested for significance applied were One way ANOVA followed by Dunn's post-hoc test for multiple comparisons, student t test. P-values $<0.05$ were considered significant. Spearman's correlation study was performed to find any association between damage score and the alteration of blood glucose level and also with cytokines response. The statistical analysis was carried out in SPSS 20.0 version.

\section{Result:-}

Antibody levels in immunized BALB/c mice:

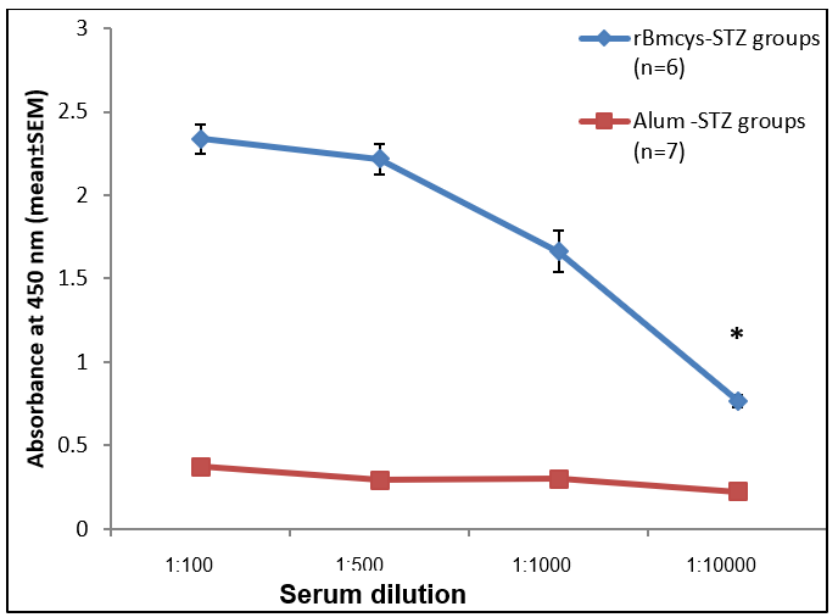

Figure 1:- Antibody levels in mice immunized with rBmCys antigen against respective antigen compared to AlumSTZ group.

BALB/C mice were immunized with $\mathrm{rBmCys}$ antigen, developed significantly high levels of anti-rBmCys $\operatorname{IgG}$ antibodies in their sera $(\mathrm{p}<0.0001)$. (Figure 1) Similarly, significantly elevated anti-Bm mf ES IgG antibodies and anti-Bm mf S IgG antibody level were found.

\section{Diabetes status in mice:-}

Mice pre-treated with rBmCys followed by induction with STZ (rBmCys-STZ) showed significantly lower fasting blood glucose levels compared to mice pre-treated with only alum adjuvant or in untreated mice. Similarly, fasting blood glucose levels of mice pretreated with $\mathrm{Bm}$ mf ES antigen and $\mathrm{Bm} \mathrm{mf} S$ antigen showed significant reduction $(\mathrm{p}<0.001)$ as compared to control group of mice (Alum-STZ). (Figure 2)

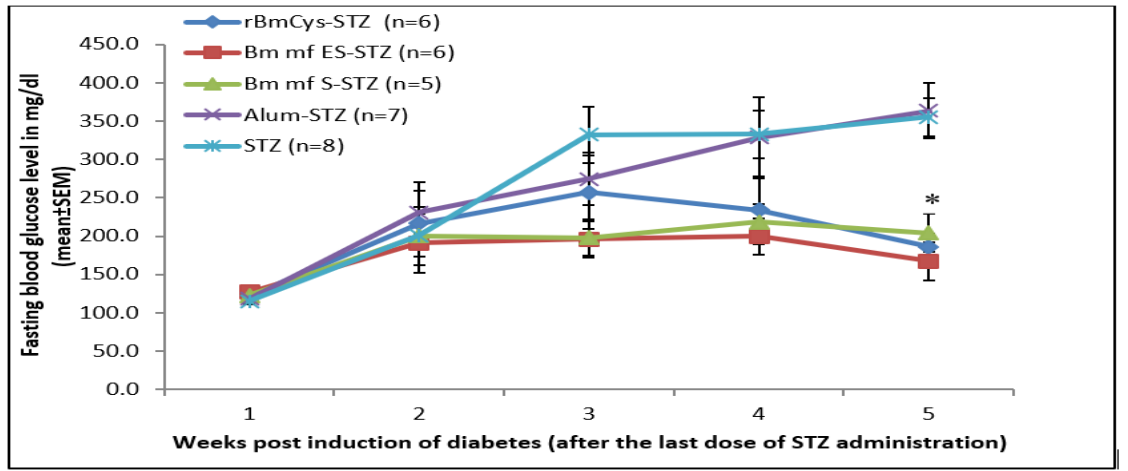

Figure 2:- Fasting blood glucose levels and diabetes status in mice pre-treated with filarial proteins 


\section{Effect on cellular proliferation:-}

Proliferative response of spleen cells from diabetic group of animals pre-treated with rBmCys (STZ-rBmCys) showed significantly pronounced splenocyte proliferation response $(\mathrm{p}<0.001)$ upon stimulation with $\mathrm{rBmCys}$ compared to the spleen cells from mice treated with alum alone (STZ-Alum).(Figure 3).

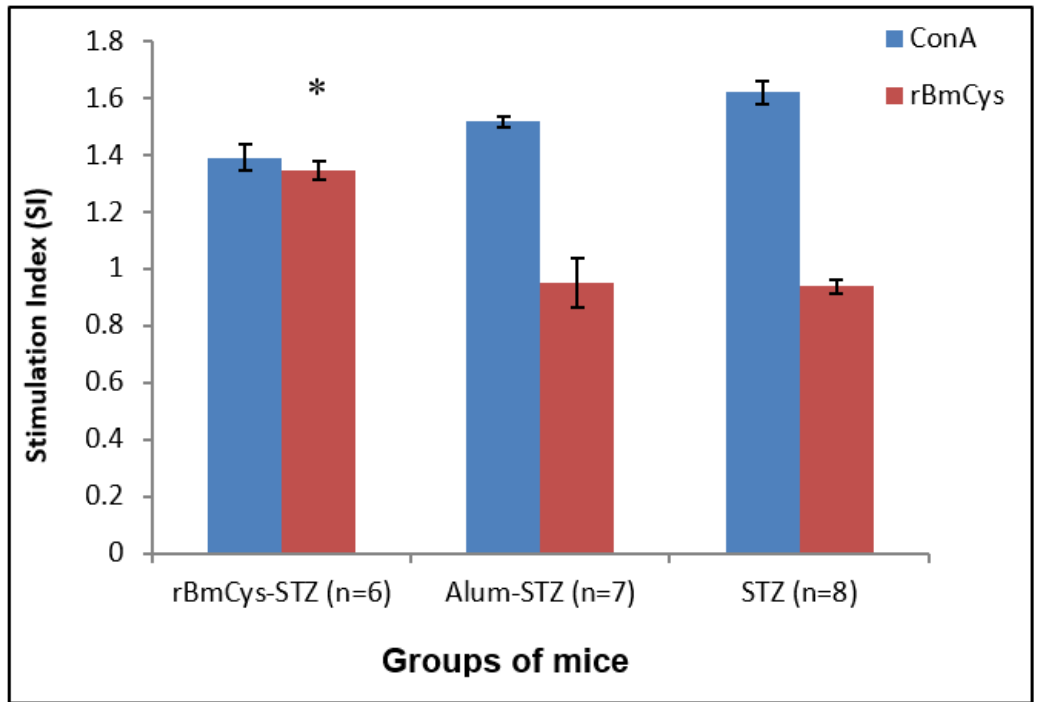

Figure 3:- Proliferation of splenocytes of STZ-induced diabetic mice pre-treated i.p. with rBmCys Similarly, upon stimulation with Bm mf ES or Bm mf S antigens, proliferative response of splenocytes of mice pre-treated with Bm $\mathrm{mf}$ ES was found to be significantly higher $(\mathrm{p}<0.0001)$ compared to that in Alum-STZ and the STZ.

\section{Analysis of cytokines in the supernatants of splenocyte cultures:-}

Splenocytes from STZ induced diabetic mice pre-treated with $\mathrm{rBmCys}$, Bm $\mathrm{mf}$ ES or Bm mf S showed significantly higher secretion of anti-inflammatory cytokine IL-10, IL-4 and reduced level of IL-5 cytokine compared to other groups. Similarly, the level of TNF- $\alpha$ and interferon- $\gamma($ IFN- $\gamma$ ) was found to be significantly lower in the splenocyte culture supernatant of $\mathrm{rBmCys}$ group, $\mathrm{Bm} \mathrm{mf} \mathrm{ES}$ or Bm mf S compared to other groups.(Figure 4-8)

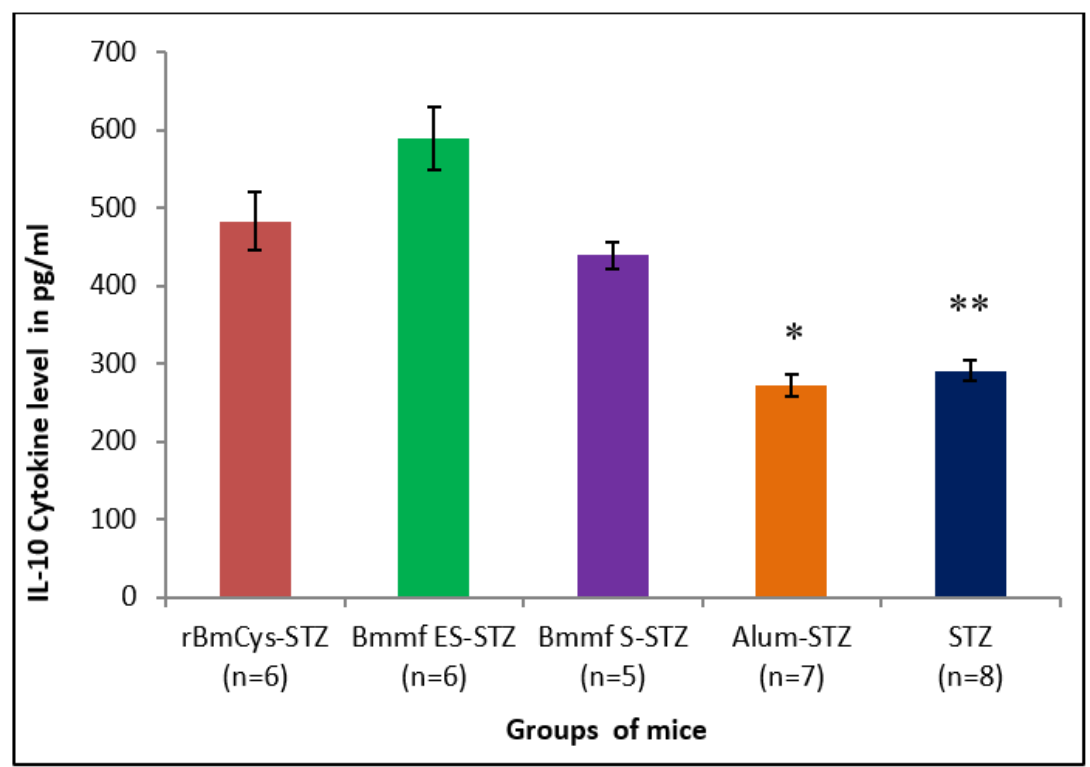

Figure 4:- Cytokine levels to stimulation with rBmCys or Bm mf ES or Bm mf S in IL-10 


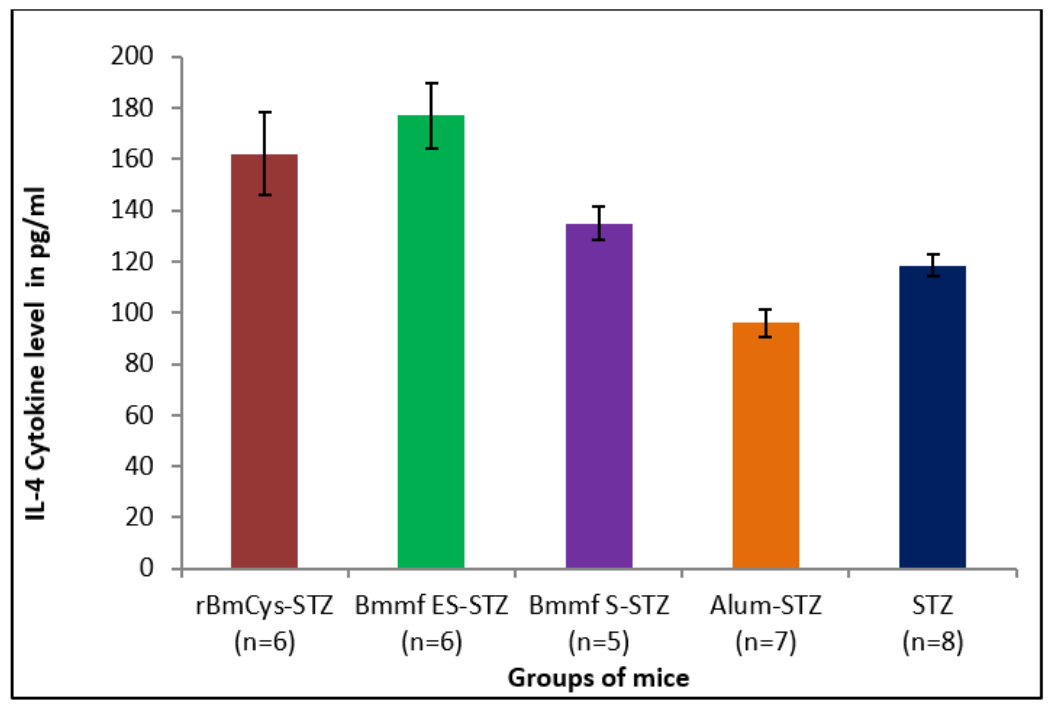

Figure 5:- Cytokine levels to stimulation with rBmCys or Bm mf ES or Bm $\mathrm{mf} S$ in IL-4

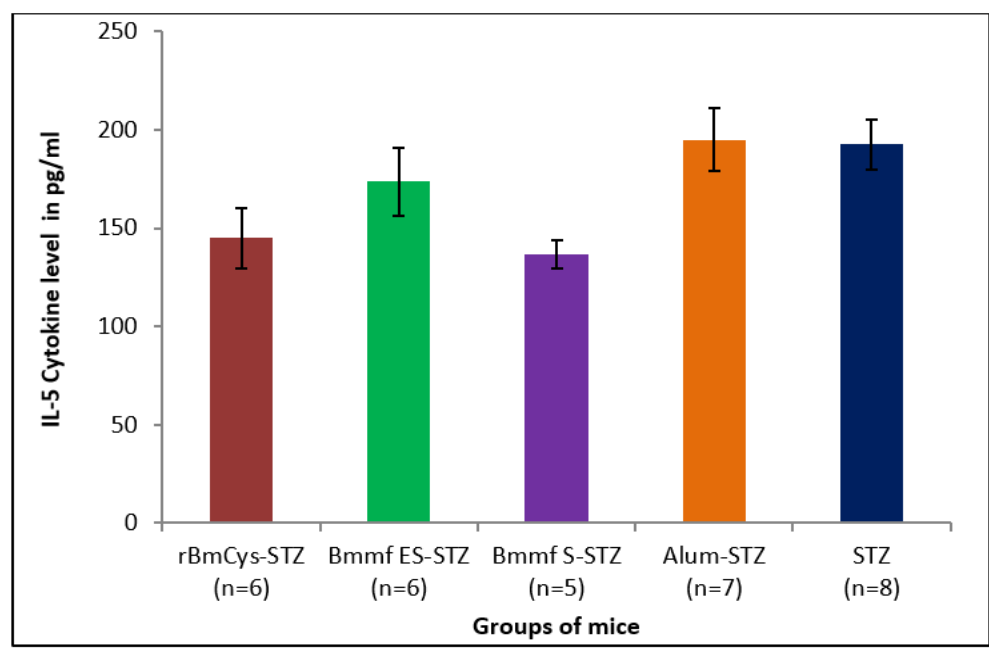

Figure 6:- Cytokine levels to stimulation with rBmCys or Bm mf ES or Bm mf S in IL-5

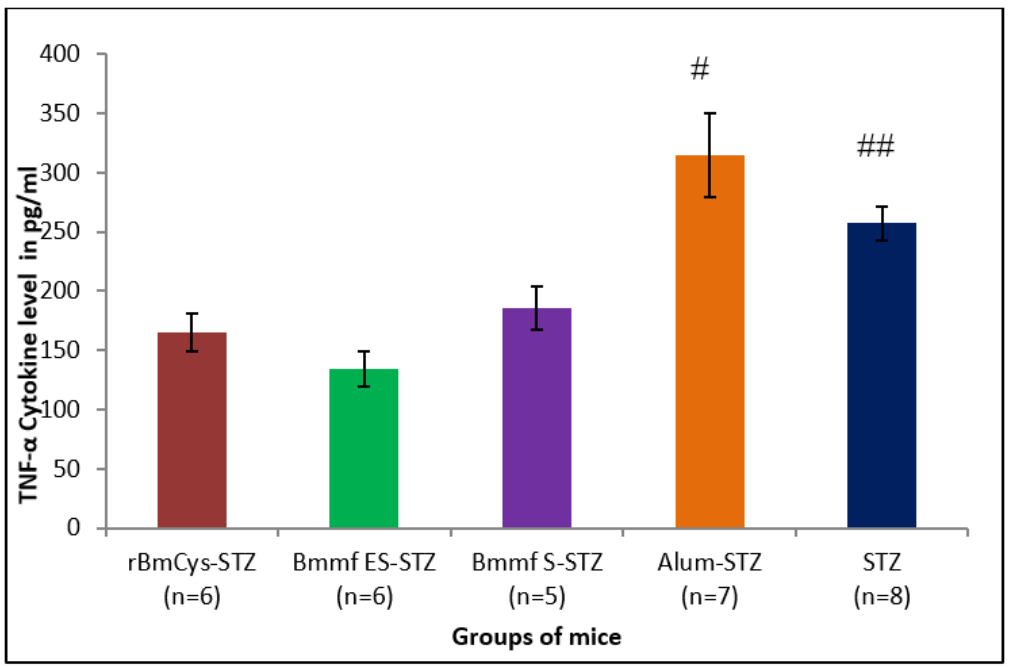

Figure 7:- Cytokine levels to stimulation with $\mathrm{rBmCys}$ or $\mathrm{Bm} \mathrm{mf} \mathrm{ES}$ or Bm $\mathrm{mf} \mathrm{S}$ in TNF- $\alpha$ 


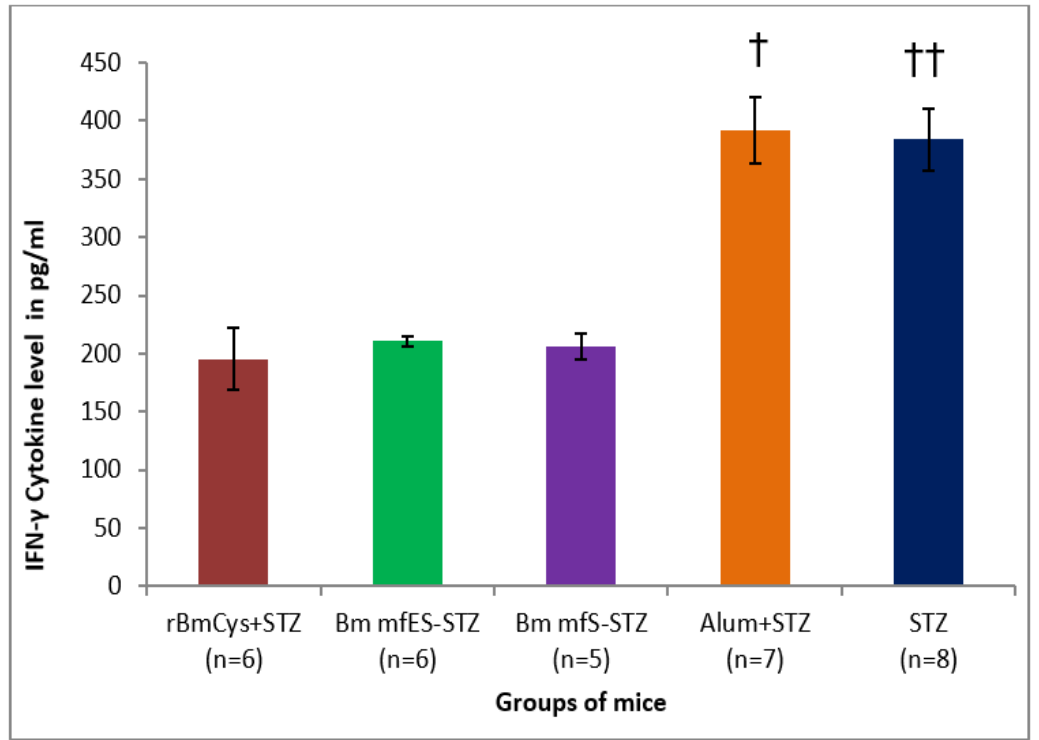

Figure 8:- Cytokine levels to stimulation with rBmCys or Bm mf ES or Bm mf S in IFN- $\gamma$

\section{Levels of anti-insulin antibodies:}

Significantly higher levels of insulin specific IgG1 antibodies were found in diabetic group of mice pre- treated with Bm mf ES compared to the levels in STZ-Alum ( $\mathrm{p}<0.002)$ or STZ (0.005) group of diabetic mice.(Figure 9) Similarly, significantly higher levels of insulin specific IgM antibodies were found in diabetic group of mice pretreated with $\mathrm{rBmCys}$ and $\mathrm{Bm} \mathrm{mf}$ ES compared to the levels in STZ-Alum.(Figure 10) In contrast, significantly lower level of anti-insulin IgG2a antibodies were found in $\mathrm{rBmCys}$ or $\mathrm{Bm} \mathrm{mf} \mathrm{ES}$ or Bm $\mathrm{mf}$ group of mice compared to the untreated STZ group of mice.

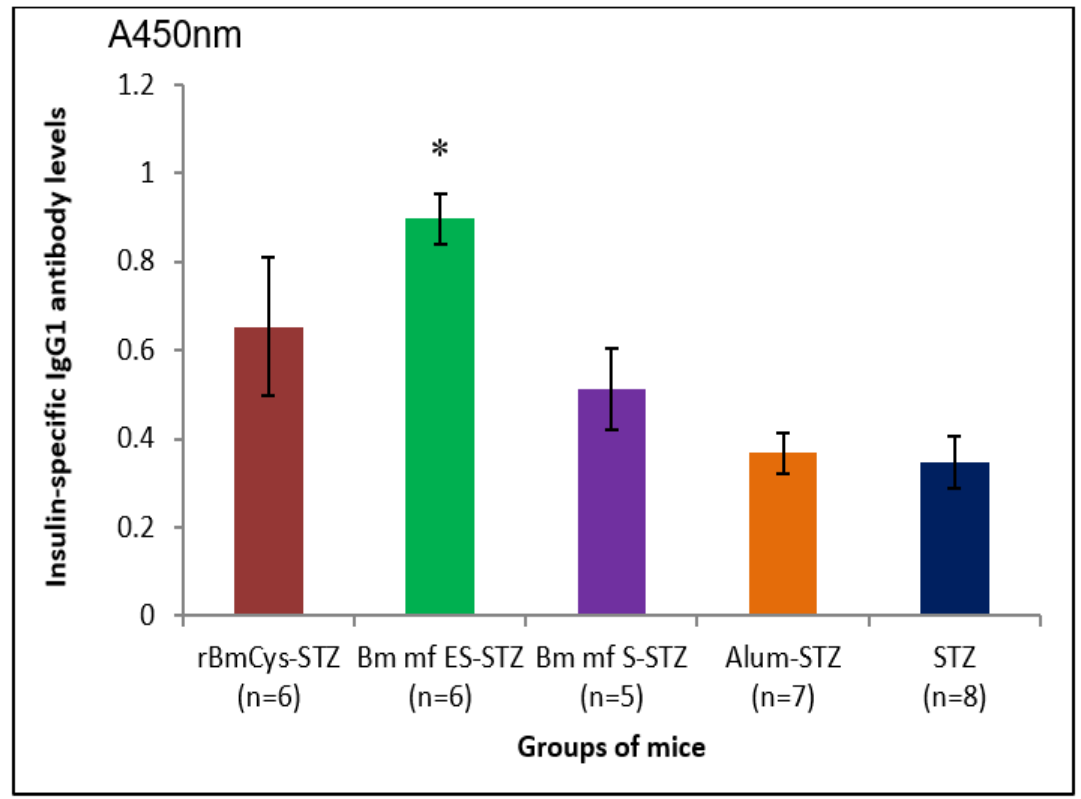

Figure 9:- Anti-insulin IgG1 auto-antibodies in mice pretreated with filarial antigen followed by the STZ induction. 


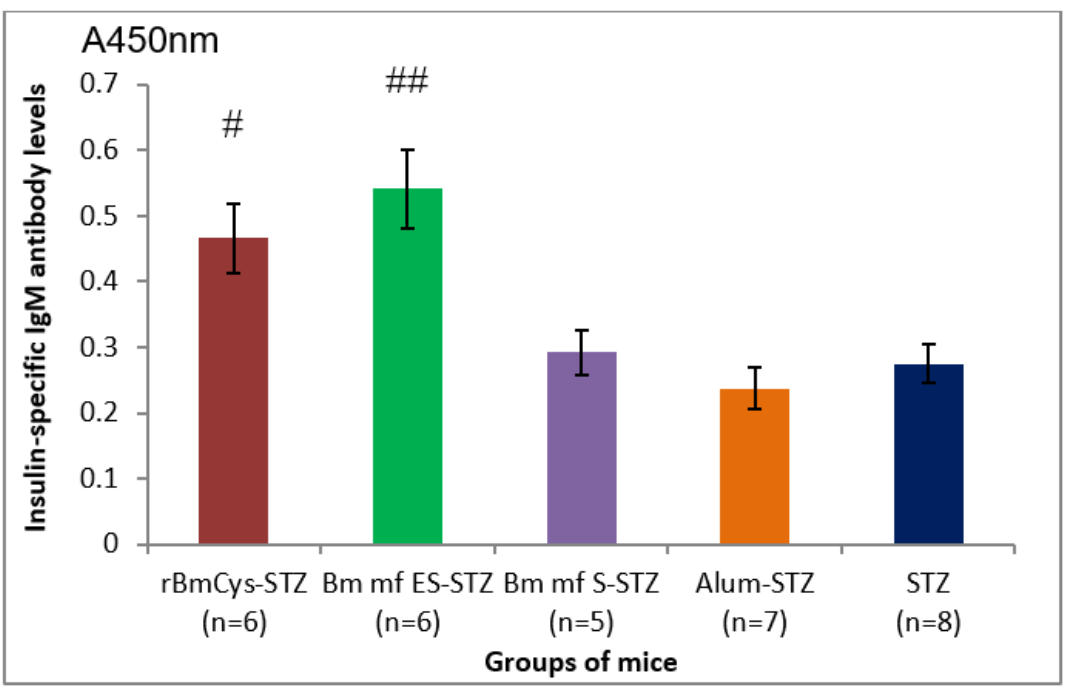

Figure 10:- Anti-insulin IgM auto-antibodies in mice pretreated with filarial antigen followed by the STZ induction

\section{Histopathological assessment of pancreas:-}

The groups of diabetic mice pre-treated with $\mathrm{rBmCys}$ showed significant number of healthy pancreatic islets cells, whereas pancreas from the diabetic groups of mice pre-treated with alum showed, as expected, either severe insulitis or complete destruction of islets.(Figure 11)

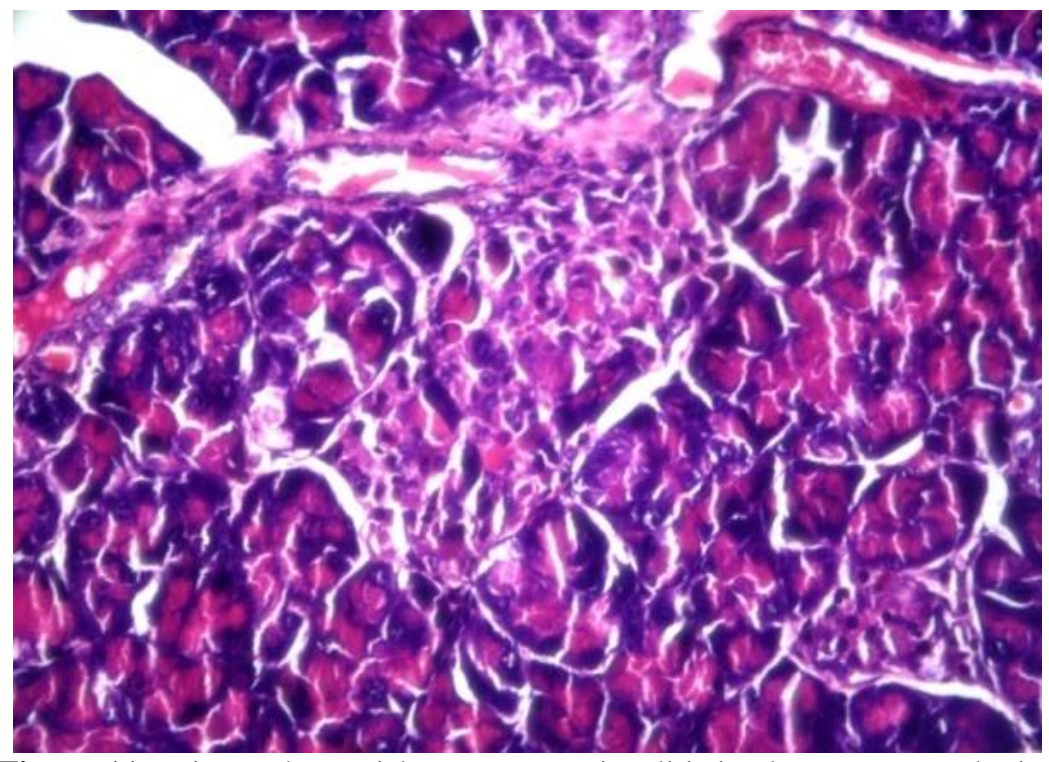

Figure 11:- Figure shows islets as severe insulitis in alum pre-treated mice

\section{Discussion:-}

Many recent epidemiologic and experimental data have shown that helminths infections are able to inhibit the development of autoimmune processes and pathology in animal experimental models and probably in humans. Immunomodulatory activity of helminth and its products have shown to ameliorate and inhibit several immunological diseases like type 1 diabetes, rheumatoid arthritis, asthma, colitis etc. in animal models. (Zaccone et al., 2003; Harnett et al., 2004). This is attributed to the ability of these parasites to manipulate and down-regulate the immune responses of their hosts. Our study provides powerful proof of the concept that the helminths specific recombinant proteins or the excretory secretory or soluble antigens can protect against autoimmunity.

In this study, we investigated the role of prophylactic treatment with recombinant Brugia malayi cystatin (rBmCys), Excretory secretory $(\mathrm{Bm} \mathrm{mf} \mathrm{ES})$ and microfilarial soluble $(\mathrm{Bm} \mathrm{mf} \mathrm{ES})$ filarial proteins for protection from the 
development of STZ (Streptozotocin) induced T1D. In this study, we found out that there was reduction in the glucose level in the mice pre-treated with filarial derived proteins. Only $42 \%$ of $\mathrm{rBmCys}$ or Bm mf S pretreated group of mice and $28 \%$ of $\mathrm{Bm}$ mf ES pretreated group of mice developed diabetes at the end of the 4th week after the last dose of STZ. Although all mice pre-treated with filarial antigens had evidence of ongoing islet cell inflammation by histology, but mice pretreated with $\mathrm{rBmCys} / \mathrm{Bm} \mathrm{mf} \mathrm{ES} / \mathrm{Bm} \mathrm{mf} \mathrm{S}$ have shown more number of healthy and non-infiltrated islets and also reduced lymphocytic infiltration resulting in reduced destruction of the pancreatic islets as compared to the alum treated mice.

The levels of IL-4, IL-10, interferon- $\gamma($ IFN- $\gamma$ ) and TNF- $\alpha$ were analyzed in the culture supernatants of splenocytes followed by stimulation with respective proteins. The findings showed pronounced splenocyte proliferation response against respective antigens in all the mice pre-treated with filarial antigens $\mathrm{rBmCys} / \mathrm{Bm} \mathrm{mf} \mathrm{ES} / \mathrm{Bm} \mathrm{mf}$. Many studies have shown that protection against the onset of diabetes by helminths or their antigens has been uniformly associated with a general Th2 shift (Zaccone et al., 2003;Imai et al., 2001). It is believed that in T1D, which is mediated by Th1 response, helminths might be skewing this islet specific immune response towards non-pathogenic Th2 response (Kullberg et al., 1992; La Flamme et al., 2002). The findings of the present study are in consistence with this concept as the filarial proteins $\mathrm{rBmCys}, \mathrm{Bm} \mathrm{mf}$ ES and $\mathrm{Bm}$ mf S seems to be mediating through antiinflammatory cytokine IL-10 to shift the immune response towards Th2 type to suppress T1D.

Hence it is likely that a range of mechanisms including those initially acting on the innate immunity and subsequently on T cell function seem to be operative in the influence of infections to suppress T1D. Thus, the results of this study has shown that, Brugia malayi cystatin (rBmCys) or Excretory secretory (Bm mf ES) or microfilarial soluble (Bm mf $\mathrm{S}$ ) antigens given during antigen priming were found to be effective in reducing the fasting plasma glucose level, pancreatic inflammation and severe destruction of $\beta$-cells of pancreatic islets.

\section{Conclusion:-}

Both genetic and environmental factors are widely recognized as features of etiological importance in the disease progression and clinical onset. One of the suggested environmental modifiers is 'infection', which is shown to play a role in preventing the onset of this autoimmune disease in rodent models and also possibly in humans. Multiple studies have found that individuals infected with chronic parasitic worm infections have lower rated of autoimmune diseases than others living in the same environment. From the findings of this study it can be envisaged that the treatment of mice with filarial antigens ( $\mathrm{BBmCys}, \mathrm{Bm} \mathrm{mf} \mathrm{ES}$ and $\mathrm{Bm} \mathrm{mf} \mathrm{S}$ ) before induction of T1D had protective effect against this autoimmune disease. This protection was found to be associated with changes in humoral, cellular and cytokine responses that reflected a shift from Th1 to Th2 type of immune response. Further studies on the dose dependent responses and long term follow up of treated animals with more effective adjuvants may help in formulating effective and novel therapeutic compounds for T1D and other autoimmune diseases.

\section{References:-}

1. Aravindhan V, Mohan V, Surendar J. Decreased prevalence of lymphatic filariasis among diabetic subjects associated with a diminished pro-inflammatory cytokine response. PLoS Negl Trop Dis. 2010; 4: e707.

2. Chenthamarakshan V, Reddy MVR, Harinath BC. Diagnostic potential of fractionated Brugia malayi microfilarial excretory-secretory antigen for bancroftian filariasis. Trans Roy Soc Trop Med \& Hyg. 1996a; 90: 250- 254.

3. Fleming JO and Cook TD. Multiple sclerosis and the hygiene hypothesis. NEUROLOGY. 2006; 67 (1-2): 208586.

4. Harnett W, Harnett MM, Byron O. Structural/functional aspects of ES-62-a secreted immunomodulatory phosphorylcholine-containing filarial nematode glycoprotein. Curr Protein Pept Sci. 2003; 4 (1): 59- 71.

5. Harnett W, McInnes IB, Harnett MM. ES-62 a filarial nematode derived immuno-modulator with antiinflammatory potential. Immunology Letters. 2004; 94 (1-2): 27- 33.

6. Hartmann S, Kyewski B, Sonnenburg B, and Lucius R. A filarial cysteine protease inhibitor down-regulates Tcell proliferation and enhances interleukin-10 production. Eur. J. Immunol. 1997; 27: 2253- 2260.

7. Hartmann S and Lucius R Modulation of host immune responses by nematode cystatins. Int. J. Parasitol. 2003; 33: $1291-1302$.

8. Hubner MP, Stocker JT and Mitre E. Inhibition of type 1 diabetes in filaria-infected non-obese diabetic mice is associated with a T helper type 2 shift and induction of FoxP3+regulatory T cells. Immunology. 2009; 127 (4): $512-522$. 
9. Imai S, Tezuka H, Furuhashi Y, Muto R, Fujita K. A factor of inducing IgE from a filarial parasite is an agonist of human CD40. J Biol Chem. 2001; 276(49): 46118- 24.

10. Kaliraj P, Ghirnikar SN, Harinath BC. Fractionation and evaluation of Wuchereria bancrofti microfilarial antigens in immunodianosis of bancroftian filariasis. Ind J Exptl Biol. 1982; 20: 440- 444.

11. Kullberg MC, Pearce EJ, Hieny SE, Sher A, Berzofsky JA. Infection with Schistosoma mansoni alters Th1/Th2 cytokine responses to a non-parasite antigen. J Immunol. 1992; 148: 3264- 70.

12. La Flamme AC, Scott P, Pearce EJ. Schistosomiasis delays lesion resolution during Leishmania major infection by impairing parasite killing by macrophages. Parasite Immunol. 2002; 24: 339- 45 .

13. Maizels RM, Pearce EJ, Artis D, Yazdanbakhsh M and Wynn TA. Regulation of pathology and immunity in helminth infections. Journal of Experimental Medicine. 2009; 206: 2059- 2066.

14. McInnes IB, Leung BP, Liew FY. A novel therapeutic approach targeting articular inflammation using the filarial nematode-derived phosphorylcholine-containing glycoprotein ES-62. Journal of Immunology. 2003; 171: 2127- 2133.

15. Onkamo P, Väänänen S, Karvonen M, Tuomilehto J. Worldwide increase in incidence of Type I diabetes the analysis of the data on published incidence trends. Diabetologia 1999; 42 (12): 1395- 1403.

16. Patel SP, Jarvelin MR, Little MP. Systematic review of worldwide variations of the prevalence of wheezing symptoms in children. Environ Health. 2008; 7: 57.

17. Ramachandran A, Snehalatha C, Krishnaswamy CV. The Incidence of IDDM in children in urban population in southern India. Diabetes Res Clin Pract. 1996; 34: 79- 82.

18. Saunders KA, Raine T, Cooke A, Lawrence CE. Inhibition of autoimmune type 1 diabetes by gastrointestinal helminth infection. Infect Immun. 2007; 75: 397- 407.

19. Schierack P, Lucius R, Sonnenburg B, Schilling K, Hartmann S. Parasite-specific immunomodulatory functions of filarial cystatin. Infect Immun. 2003; 71 (5): 2422- 9.

20. Sewell DL, Reinke EK, Hogan LH, Sandor M, Fabry Z. Immunoregulation of CNS autoimmunity by helminth and mycobacterial infections. Immunol Lett. 2002; 82 (1-2):101- 10.

21. Tisch R and McDevitt H. Insulin dependent Diabetes Mellitus. Cell. 1996; 85291- 297.

22. Weinstock JV, Elliot DE. Helminths and the IBD hygiene hypothesis. Inflamm Bowel Dis. 2009; 15: 128- 33.

23. Zaccone P, Fehervari Z, Jones FM, Sidobre S, Kronenberg M, Dunne DW, Cooke A. Schistosoma mansoni antigens modulate the activity of the innate immune response and prevent onset of type 1 diabetes. Eur. J. Immunol. 2003; 33: 1439- 1449. 